\title{
Improving genetic transformation of European chestnut and cryopreservation of transgenic lines
}

\author{
E. Corredoira · M.C. San-José · A.M. Vieitez · A. Ballester ${ }^{1}$ \\ Instituto de Investigaciones Agrobiológicas de Galicia, CSIC \\ Avenida de Vigo, s/n, Apartado 122 \\ 15080 Santiago de Compostela, Spain \\ e-mail: aballester@,iiag.cesga.es \\ Tel: + 34981590958 \\ Fax: + 34981592504 \\ ${ }^{1}$ Corresponding author
}

\begin{abstract}
The aim of the present work was to study the effect of the developmental stage of the somatic embryos and of the genotype on the genetic transformation of embryogenic lines of European chestnut (Castanea sativa Mill.) and the cryopreservation of the embryogenic lines that are generated. As an initial source of explants in the transformation experiments, it was found that the use of somatic embryos isolated in the globular stage or clumps of 2-3 embryos in globular/ heartshaped stages was more effective $(30 \%)$ than when embryos at the cotyledonary stage were used (6.7\%). All of the seven genotypes tested were transformed, and transformation efficiency was clearly genotype dependent. Three transgenic lines were successfully cryopreserved using the vitrification procedure, and the stable integration of the uidA gene into the transgenic chestnut plants that were regenerated subsequent to cryopreservation was demonstrated.
\end{abstract}

Keywords Agrobacterium $\cdot$ Castanea sativa $\cdot$ Embryogenic lines

Abbreviations BA: 6-benzyladenine $D M S O$ : dimethylsulfoxide $\cdot M S$ : Murashige and Skoog medium $\cdot N A A$ : $\alpha$-Naphthaleneacetic acid $\cdot$ PVS2: Plant Vitrification Solution 2 


\section{Introduction}

European chestnut (Castanea sativa Mill.) is a tree species with a wide distribution, as it is present in 25 European countries, covering an area of over 2 million hectares (Conedera et al. 2004). Considered a forest tree, chestnut is also used in agriculture as a cultivated crop and, in Europe, it is managed as high forest, coppices and orchards. In addition to its original productive role, European chestnut stands are playing important roles in wild life conservation, protection from erosion, landscape, rural tourism and recreational areas.

The root-rot disease (caused by Phytophthora spp.) and chestnut blight (caused by Cryphonectria parasitica) are the two most important diseases that affect not only the European chestnut but also the American chestnut (C. dentata). Conventional breeding programmes making use of the disease resistance/tolerance shown by the Asian chestnut species (C. crenata, C. mollissima) requires the screening of large numbers of progeny over several generations. Consequently, with the long periods of time required for these programmes, only first generation Euro-Asian hybrids (in which some of the specific traits of European chestnut are lost) are widespread in several European countries. Alternatively, genes related to fungal disease resistance of potential use in chestnut restoration have been identified in both Europe (Collada et al. 1992; García Casado et al. 2000) and America (Powell et al., 1995; Liang et al., 2002). Although these genes have not yet been expressed in chestnut, the first attempts to develop genetic transformation protocols in both European (Corredoira et al. 2004a) and American (Polin et al. 2006; Rothrock et al. 2007) species have recently been published. In European chestnut, plant regeneration through the induction of somatic embryogenesis is well established, not only from juvenile material of unknown genetic value, such as immature zygotic embryos, but also from mature clonal material (Corredoira et al. 2003, 2006). These embryogenic systems allowed the definition of the first protocol for genetic transformation of European chestnut (Corredoira et al. 2004a). In this protocol, parameters such as the bacterial strain/plasmid combination, the effect of acetosyringone, the cell strain density and the selection procedure, were initially evaluated having used one single embryogenic line. Optimized protocols are required and, in this respect, a number of factors including the developmental stage of the somatic embryos to be used as target explants, and the genotype, need to be evaluated in order to improve the transformation efficiency 
Due to the long cycles needed in the forest tree breeding programmes, cryopreservation (i.e. the storage of material in liquid nitrogen) may be a powerful tool during progeny testing. Moreover, this technique is generally used for the long-term maintenance of specific genotypes or cell lines or to avoid the risk of possible somaclonal variation as a consequence of prolonged tissue culture management.

Cryopreservation protocols of embryogenic cultures have been developed for several woody plants (Martínez et al. 2003; Valladares et al. 2004; Lambardi et al. 2000) and in recent years use has been made of the vitrification method, which involves the dehydration of samples with highly concentrated vitrification solutions followed by rapid cooling (Sakai, 2000). According to this procedure, the successful cryopreservation of in vitro-grown shoot tips and somatic embryos of European chestnut has been reported (Corredoira et al. 2004b; Vidal et al. 2005). Studies on the cryopreservation of transgenic lines in hardwood trees are scarce, and no references have been found for transgenic chestnut. Ryyänen et al. (2002) developed a cryopreservation protocol for transgenic silver birch lines using vegetative buds as material for cryopreservation. In addition, Jokipii et al. (2004) showed that it was possible to apply cryopreservation techniques to the preservation of valuable clones and transgenic lines of aspen, using both in vitro shoot cultures and in vivo plants.

The aims of the present work are i) to study the effects of the developmental stage of the somatic embryos and the genotype on chestnut transformation efficiency; and ii) to evaluate the capacity for cryopreservation and further plant recovery of chestnut transgenic embryogenic lines.

\section{Material and methods}

Plant material and culture conditions

Seven embryogenic lines were used in this study. Line C12-H1 was initiated from leaf explants isolated from shoot multiplication cultures of Castanea sativa Mill. (Corredoira et al. 2003, 2006). Embryogenic lines CI-1, CI-3, CI-6, CI-7 and CI-9 were induced from zygotic embryos of C. sativa. Embryogenic line HV-Z2 derives from zygotic embryos of a Castanea sativa x C. crenata hybrid (Vieitez 1995; Corredoira et al. 2006). 
Regardless of the type of assay, the embryogenic cultures were routinely maintained by secondary embryogenesis with sequential subcultures at 6-week intervals on proliferation medium consisting of MS (Murashige and Skoog, 1962) half-strength macronutrients, vitamins, $3 \mathrm{mM}$ glutamine, $0.1 \mathrm{mg} / \mathrm{l} \mathrm{BA}, 0.1 \mathrm{mg} / 1 \mathrm{NAA}, 3 \%$ sucrose and $0.7 \%$ Sigma agar. The $\mathrm{pH}$ of the media was adjusted to 5.7 before autoclaving at $120^{\circ} \mathrm{C}$ for $20 \mathrm{~min}$. The cultures were incubated at $25^{\circ} / 20^{\circ} \mathrm{C}$ (day/night) under a $16 / 8 \mathrm{~h}$ photoperiod and a photon flux density of 50-60 $\mu \mathrm{molm}^{-2} \mathrm{~s}^{-1}$, considered as the standard conditions (Corredoira et al. 2003).

Bacterial strain and plasmid

Agrobacterium tumefaciens strain EHA105 (Hood et al., 1993) carrying the plasmid pUbiGUSINT (Humara et al., 1999) was used for transformation experiments. Among others studied, this combination has been found to be the most suitable for European chestnut transformation (Corredoira et al., 2004). The binary vector contains the neomycin phosphotranspherase II (nptII) marker gene driven by the nos promoter for kanamycin selection and the $\beta$-glucuronidase (GUS) reporter uidA gene driven by the maize ubiquitin (UBI-I) promoter. The plasmid carries the GUS gene with an intron that prevents its expression in Agrobacterium. Bacteria cultures were maintained and used according to Corredoira et al. (2004a).

Transformation, selection and molecular analyses

Isolated somatic embryos at different developmental stages were used in the different experiments performed. Plant material isolated from 4-week-old cultures was immersed for $30 \mathrm{~min}$ in a bacterial suspension grown up to an $\mathrm{OD}_{600}=0.6$, blot-dried on sterile filter paper and transferred to proliferation medium. After 4 days of co-culture in the dark at $25^{\circ} \mathrm{C}$, the plant material was washed for $30 \mathrm{~min}$ with sterilized distilled water containing $500 \mathrm{mg} / \mathrm{l}$ cefotaxime and transferred to Petri dishes containing proliferation medium supplemented with $300 \mathrm{mg} / \mathrm{l}$ carbenicillin, $200 \mathrm{mg} / \mathrm{l}$ cefotaxime and $150 \mathrm{mg} / \mathrm{l}$ kanamycin as selection medium.

After 8 weeks of culture with subculture to fresh selection medium at 15-day intervals, kanamycin-resistant embryos were transferred to fresh selective medium for a further 4 weeks. At the end of this period, kanamycin-resistant embryos were recorded 
and surviving explants were also evaluated on the basis of GUS activity (Jefferson, 1987) to determine the transformation efficiency, defined as the percentage of initial explants that developed GUS-positive embryogenic cultures. To confirm the presence of the nptII and uidA genes in GUS-positive embryogenic lines, PCR and Southern-blot methods were used and developed as described by Corredoira et al. (2004a).

Effect of explant type and genotype

To study the effect of the type of explant, isolated globular somatic embryos (1-2 $\mathrm{mm})$, clumps of 2-3 globular/heart-shaped embryos $(2-5 \mathrm{~mm}, 6-8 \mathrm{mg})$ or isolated cotyledonary embryos $(3-5 \mathrm{~mm})$ were tested using the embryogenic line CI-7.

Seven embryogenic lines (C12-H1, CI-1, CI-3, CI-6, CI-7, CI-9 nad HV-Z2) were used to study the effect of the genotype on the transformation efficiency. Clumps of 2-3 globular/heart-shaped embryos were used in this experiment.

These parameters were evaluated on the basis of the GUS activity (transformation efficiency) of the somatic embryos, and in each experiment six replicates were used per treatment, each containing ten somatic embryos.

Cryopreservation of transgenic lines

Somatic embryos of C. sativa were collected from 3 transgenic lines and the corresponding C12-H1 wild-type line, as shown in Table 1. The plasmids used in the transformation experiments were pBI121, pUbiGUSINT and p35SGUSINT, all of which contain the nptII marker gene and the reporter uidA gene, under the nopaline synthase (nos), the maize ubiquitin (Ubi) and the CaMV $35 S$ promoters, respectively

Cryopreservation was performed using the vitrification-based procedure (Engelmann 2004). Briefly, the transgenic embryogenic cultures were subcultured at six-week intervals onto selection medium and the wild-type line was maintained on proliferation medium as previously described. The somatic embryo clumps $(2-5 \mathrm{~mm}, 6-8 \mathrm{mg}$, clumps of globular/heart-shaped somatic embryos) were pre-cultured for 3 days on proliferation medium (without glutamine and plant growth regulators) containing $0.3 \mathrm{M}$ sucrose and were then placed, 10 explants/vial, in $2 \mathrm{ml}$ cryovials with $1.8 \mathrm{ml}$ of PVS2 vitrification solution consisting of $30 \% \mathrm{w} / \mathrm{v}$ glycerol, $15 \% \mathrm{w} / \mathrm{v}$ DMSO, $15 \% \mathrm{w} / \mathrm{v}$ ethylene glycol in MS medium containing 0.4 M sucrose (Sakai et al. 1990). After $60 \mathrm{~min}$ at $0^{\circ} \mathrm{C}$ in PVS2 
solution, half of the samples were re-suspended in $0.6 \mathrm{ml}$ of the PVS2 solution and then immersed in LN. After $24 \mathrm{~h}$ they were removed from the LN and thawed by placing the vials in a water bath at $40^{\circ} \mathrm{C}$ for $2 \mathrm{~min}$. Untreated controls received neither sucrose preculture nor PVS2 treatment and half of them were also immersed in LN. All somatic embryo clumps (treated and untreated with PVS2, with and without immersion in LN) were cultured in proliferation medium, where they remained until they were evaluated 6 weeks later (Corredoira et al. 2004b). Embryo recovery was assessed as the percentage of explants that showed resumption of the embryogenic process.

At least three ten-clump replicates per treatment and line were used in each experiment, and each experiment was performed three times.

Stability of the inserted genes after cryopreservation was evaluated by PCR and Southern Blot analysis as previously stated (Corredoira et al. 2004a).

Statistical analyses

The results obtained in the different experiments were subjected to analysis of variance followed by mean comparison using the least significant difference (LSD) test at the $p=$ 0.05. Percentage data were subjected to arcsine transformation prior to analysis. Nontransformed data are presented in tables and figures.

\section{Results}

Effect of explant type and genotype

In this experiment, the influence of the histodifferentiation stage of the somatic embryos was evaluated by comparing isolated globular embryos with cotyledonary embryos. Clumps of 2-3 globular/heart-shaped embryos were also included as theoretically they provide a higher number of target cells to be transformed. The type of explant selected for the transformation experiments clearly influenced the efficiency of the process (Fig. 1). Although the number of potential cells to be transformed is higher in clump explants, no significant differences were found in the transformation efficiency between clumps and isolated globular embryos (in both cases, 30.0\%), while the values recorded for cotyledonary embryos were significantly lower (6.7\%). Similar results have been observed working with other embryogenic lines (data not shown). Although 
there is no significant differences in transformation efficiency between globular embryos and clumps, the use of clumps is highly recommended, as they are more easily isolated from the proliferating cultures than somatic embryos at the globular stage. According to our data, the low transformation frequency of cotyledonary embryos may be due to the low proliferation exhibited by these explants before transformation (3 somatic embryos/explant) in comparison to the untransformed clumps and globular embryos (9.2 and 9.0 somatic embryos/explant, respectively).

Seven embryogenic lines, originated from different sources, were tested for their genetic transformation capacity. Great differences in both the percentage of kanamycinresistant explants and transformation efficiency were found between the different genotypes tested (Table 2). Apparently, these differences could not be related to the origin of the line: within those lines that originated from immature zygotic embryos of C. sativa (lines CI-1, CI-3, CI-6, CI-7, and CI-9), the percentage of kanamycin-resistant explants ranged from $1.7 \%$ (line CI-6) to $41.7 \%$ (line CI-7). The transformation efficiency was, in most cases, lower than the frequencies for the kanamycin-resistant explants and varied from the $1.7 \%$ for line CI- 6 to $33.8 \%$ from line CI- 7 . In the line C12-H1 of C. sativa, that had originated from leaf pieces of in vitro cultures, GUS was expresed in $21.7 \%$ of somatic embryos, while the line HV-Z2, that had originated from immature zygotic embryos of a hybrid C. sativa $x$ C. crenata, only $1.7 \%$ of GUS positive embryos were recorded. Regardless of the percentage of transformation achieved, all lines were transformed and all of them remained embryogenic after the process. Successful proliferation of somatic embryos via secondary embryogenesis was carried out, and was independent of the transgenic line used.

Molecular analyses, germination and plantlet conversion

In these experiments, all GUS-positive embryogenic lines analyzed by PCR (64 out 195) showed the fragments corresponding to the nptII and uidA genes. The presence of T-DNA in the genome of the putative transgenic lines was also confirmed by Southern blot analysis (data not shown). In addition, transformed embryos derived from these experiments were induced to mature and germinate, as described by Corredoira et al. (2003). Low plantlet conversion rates (6.3-11.0\%) were recorded, but germinating embryos with only shoot or root development were also produced. The shoots that developed were successfully multiplied by axillary shoot proliferation (Sánchez et al., 
1997), which allowed the production of large quantities of transgenic shoots to be rooted, and rooting frequencies of $35-50 \%$ were obtained according to the different transgenic embryogenic lines tested. Transgenic chestnut plants were acclimatized in a phytotron and grown in greenhouse, and the presence of transferred genes in leaves from these plants was also verified by the GUS assay.

Cryopreservation of transgenic lines

Embryo recovery frequencies in untreated control (Fig. 2) were similar in the wild type and the three transgenic lines, with values ranging between $98 \%$ and $100 \%$. These values were not significantly reduced by the vitrification solution treatment, indicating that the PVS2 solution has no detrimental effect on embryo recovery in the lines tested (Figs. 2 LN-). When stored in LN, untreated controls either died or retained only minimal embryogenesis levels of $1.1 \%$ (data not shown). On the contrary, the embryo recovery rates of PVS2-treated and cryopreserved embryos ranged from 52 to $66 \%$ (Figs. $2 \mathrm{LN}+$ ). No differences were noted in the response among the different embryogenic lines assayed.

In both wild-type and transgenic lines new somatic embryos developed 3-4 weeks after thawing and no morphological differences or growth patterns were observed between the lines assayed. Cryopreserved transgenic embryos were subjected to maturation and germination, obtaining both plantlet conversion and plantlet regeneration through axillary shoot multiplication and rooting with similar rates to those reported in the previous section (Fig. 3).

The stability of the genes nptII and uidA in the four embryogenic lines was assessed six weeks after thawing (the non-transformed embryogenic line was used as a negative control). Two fragments of approximately 472 and 589 bp corresponding to nptII and uidA, respectively, were obtained from the DNA extracted from non-cryopreserved, vitrified somatic embryos and from vitrified embryos subjected to liquid nitrogen. The presence of uidA gene was confirmed by Southern blot analysis (Fig. 4).

For the first time, it has been demonstrated that the cryostorage of European chestnut transformed somatic embryogenic lines is possible, as the protocol defined provided succesful recuperation of the embryogenic capacity, the recovery of viable plants as well as the stability of the foreign genes inserted. 


\section{Discussion}

The genotype and the initial explant type are shown to play a crucial role in successful chestnut transformation. Differences in transformation efficiency related with the genotype were also published for other species, such as pine (Bergmann and Stomp, 1992), aspen (Fladung et al. 1997), eucalyptus (Mullins et al. 1997) and rice (Lin and Zhang, 2005). In American chestnut (Polin et al. 2006), five of the six genotypes assayed were transformed, but only four of them maintained proliferation capacity after transformation. The results published in the present work show that it was possible to transform all the European chestnut genotypes that were assayed. The most efficient lines (C12-H1 and CI-7) were also those that showed the highest proliferation capacity prior to transformation (Corredoira et al., 2003), which seems to suggest that, initially, there is a positive correlation between the proliferation capacity of the embryogenic lines and their transformation efficiency. This hypothesis, however, does not seem to be completely true, since the HV-Z2 line displayed a high proliferation capacity but low transformation efficiency (1.7\%). Lin and Zhang (2005) also determined that the average transformation efficiency of four indica rice cultivars did not correspond with the regeneration frequencies observed in experiments with non-transformed plants.

The type of initial explant is of paramount importance for successful chestnut transformation, given that higher transformation rates are obtained for embryos in the globular stage than for those in the cotyledonary stage. Bergmann and Stomp (1992) found a rapid stage of cellular division to be an even more decisive factor than genotype in the Agrobacterium-mediated transformation of Pinus radiata, and Wenck et al. (1999) also employed rapidly dividing embryogenic suspension cultures for the transformation of Norway spruce and loblolly pine. In addition, Yeung (1995) showed that somatic embryos in globular and early torpedo stages have a greater number of active embryogenic cells than embryos at the cotyledonary stage. By way of conclusion, a combination of the state of the initial explant and of the genotype should be evaluated carefully in order to improve the efficiency of the process, as has been demonstrated in European chestnut.

References to the application of cryopreservation in hardwood species are scarce in the literature, and to the best of our knowledge, are limited to sweetgum (Vendrame et al., 2001), silver birch (Ryynänen et al., 2002), and hybrid aspen (Jokipii et al., 2004). Only in sweetgum was the cryopreservation of transgenic lines not possible. 
Of the different cryopreservation procedures available, for European chestnut we have opted to use the vitrification method, as, in previous studies, we have demonstrated that it is more efficient than the desiccation method (Corredoira et al., 2004b). The behaviour of cryopreserved, transformed embryogenic lines of chestnut is similar to that of cryopreserved, non-transformed lines. Applying the vitrification method implies the use of cryoprotectors that, in certain cases (Aronen et al., 1999), may give rise to genetic aberrations. As has already been mentioned, it seems that subsequent to cryopreservation, the phenotype of the transgenic chestnut plant remains unaltered, although its genetic stability has yet to be determined by means of molecular markers. These techniques have been used in Pinus sylvestris (Häggman et al. 1998), Abies cephalonica (Aronen et al. 1999), and Populus tremula $x$ tremuloides (Jokipii et al. 2004), among other forest species. In none of these were sporadic mutations or genetic reordering detected, taking into account that the most widely used cryoprotector, DMSO, can give rise to genetic alterations in Abies cephalonica (Aronen et al. 1999) which were only detected in cultures that had not been treated with LN, suggesting that mutated cells are probably eliminated during cryostorage.

In conclusion, it can be said that the results obtained show that the genetic transformation of the European chestnut is possible by means of a well defined protocol; that this protocol can be applied to different genotypes through the use of embryogenic lines of different origins; and that, using the vitrification method, the longterm preservation in liquid nitrogen of the transformed embryogenic lines is also possible.

Acknowledgements The study was funded by the Ministerio de Educación y Ciencia (Spain) through the project AGL2005-00709. 


\section{References}

Aronen TS, Krajnakova J, Häggman HM, Ryynänen, LA (1999) Genetic fidelity of cryopreserved embryogenic cultures of open-pollinated Abies cephalonica. Plant Sci 142: $163-172$

Bergmann BA, Stomp A-M (1992) Effect of host plant genotype and growth rate on Agrobacterium tumefaciens-mediated gall formation in Pinus radiata. Phytopathol 82: $1457-1462$

Collada C, Casado C, Fraile A, Aragoncillo C (1992) Basic endochitinases are major proteins in Castanea sativa cotyledons. Plant Physiol 100: 778-783

Conedera M, Manetti MC, Giudici F, Amorini E (2004) Distribution and economic potential of the sweet chestnut (Castanea sativa Mill.) in Europe. Ecol Medit 30: 4761

Corredoira E, Ballester A, Vieitez AM (2003) Proliferation, maturation and germination of Castanea sativa Mill. somatic embryos originated from leaf explants. Ann Bot 92: $129-136$

Corredoira E, Ballester A, Vieitez FJ, Vieitez AM (2006) Somatic embryogenesis in chestnut. In: Mujib A, Samaj J (eds) Plant Cell Monogr (2) Somatic Embryogenesis., Springer, Berlin Heidelbeerg New York, pp 177-199

Corredoira E, Montenegro D, San-José MC, Vieitez AM, Ballester, A (2004a) Agrobacterium-mediated transformation of European chestnut embryogenic cultures. Plant Cell Rep 23: 311-318

Corredoira E, San-José MC, Ballester A, Vieitez AM (2004b) Cryopreservation of zygotic embryo axes and somatic embryos of European chestnut. CryoLetters 25: 3342

Engelmann F (2004) Plant cryopreservation: progress and prsopects. In Vitro Cell Dev Biol - Plant 40: 427-433

Fladung M, Kumar S, Ahuja R (1997) Genetic transformation of Populus genotypes with different chimaeric gene constructs: transformation efficiency and molecular analysis. Trans Res 6: 111-121

García-Casado G, Collada C, Allona I, Soto A, Casado R, Rodríguez-Cerezo E, Gomez L, Aragoncillo C (2000). Characterization of an aploplastic basic thaumatin-like protein from recalcitrant chestnut seeds. Physiol Plant 110:172-180 
Häggman HM, Ryynänen L, Aronen J, Krajnakova J (1998) Cryopreservation of embryogenic cultures of Scots pine. Plant Cell Tissue Organ Cult 54: 45-53

Hood EE, Gelvin SB, Melchers LS, Hoekema A (1993) New Agrobacterium helper plasmids for gene transfer to plants. Transgenic Res 2: 208-218

Humara JM, Marín MS, Parra F, Ordás RJ (1999) Improved efficiency of uidA gene transfer in stone pine (Pinus pinea) cotyledons using a modified binary vector. Can J For Res 29: 1627-1632

Jefferson RA (1987) Assaying chimeric genes in plants: the GUS gene fusion system. Plant Mol Biol Rep 5: 387-405

Jokipii S, Ryynänen L, Kallio PT, Aronen T, Häggman H (2004) A cryopreservation method maintaining the genetic fidelity of a model forest tree, Populus tremula $x$ Populus tremuloides Michx. Plant Sci 166: 799-806

Lambardi M, Fabbri A, Caccavale A (2000) Cryopreservation of white poplar (Populus alba L.) by vitrification of in vitro-grown shoot tips. Plant Cell Rep 19: 213-218

Liang H, Catramis CM, Maynard CA, Powell WA (2002) Enhanced resistance to the poplar pathogen, Septoria musiva, in hybrid poplar clones transformed with genes encoding antimicrobial peptides. Biotechnol Lett 24: 383-389

Lin YJ, Zhang Q (2005) Optimising the tissue culture conditions for high efficiency transformation of indica rice. Plant Cell Rep 23: 540-547

Martínez MT, Ballester A, Vieitez AM (2003) Cryopreservation of embryogenic cultures of Quercus robur using desiccation and vitrification procedures. Cryobiology 46: 182-189

Mullins KV, Llewellyn DJ, Hartney VJ, Strauss S, Dennis ES (1997) Regeneration and transformation of Eucalyptus camaldulensis. Plant Cell Rep 16: 787-791

Murashige T, Skoog F (1962) A revised medium for rapid growth and bioassays with tobbaco tissue cultures. Physiol Plant 15: 473-479

Polin LD, Liang H, Rothrock RE, Nishii M, Diehl DL, Newhouse AE, Nairn CJ, Powell WA, Maynard CA (2006) Agrobacterium-mediated transformation of American chestnut (Castanea dentata (Marsh.) Borkh.) somatic embryos. Plant Cell Tiss Org Cult 84: 69-78

Powell WA, Catranis CM, Maynard CA (1995) Synthetic antimicrobial peptide design. Mol Plant Microbe Interact 8: 792-794 
Rothrock RE, Polin-McGuigan LD, Newhouse AE, Powell WA, Maynard CA (2007) Plate flooding as an alternative Agrobacterium-mediated transformation method for American chestnut somatic embryos. Plant Cell Tissue Org Cult 88: 93-99

Ryynänen L, Sillanpää M, Kontunen-Soppela S, Timonen H, Kangasjärvi J, Vapaavuori E, Häggman H (2002) Preservation of transgenic silver birch (Betula pendula Roth) lines by means of cryopreservation. Mol Breeding 10: 143-152

Sakai A, Kobayashi S, Oiyama I (1990) Cryopreservation of nucellar cells of navel orange (Citrus sinensis Osb. var. brasiliensis Tanaka) by vitrification. Plant Cell Rep 9: $30-33$

Sakai A (2000) Development of cryopreservation techniques. In: Engelmann F, Takagi $\mathrm{H}$ (eds) Cryopreservation of tropical plant germplasm. Tsukuba: JIRCAS; Rome: IPGRI, pp 1-7.

Sánchez MC, San-José MC, Ferro F, Ballester A, Vieitez AM (1997) Improvimg micropropagation conditions for adult-phase shoots of chestnut. J. Horti Sci 72: 433443

Valladares S, Toribio M, Celestino C, Vieitez AM (2004) Cryopreservation of embryogenic cultures from mature Quercus suber trees using vitrification. CryoLetters 25: 177-186

Vendrame WA, Holliday CP, Montello PM, Smith DR, Merkle SA (2001) Cryopreservation of yellow-poplar and sweetgum embryogenic cultures. New For 21: 283-292

Vidal N, Sánchez C, Jorquera L, Ballester A Vieitez AM (2005) Cryopreservation of chestnut by vitrification of in vitro-grown shoot tips. In Vitro Cell Dev Biol-Plant 41: 63-68

Vieitez FJ (1995) Somatic embryogenesis in chestnut. In: Jain S, Gupta P, Newton R (eds.) Somatic Embryogenesis in Woody Plants, Vol. 2. Kluwer Academic Publishers, The Netherlands, pp 375-407

Wenck AR, Quinn M, Whetten RW, Pullmann G, Sederoff R (1999) High-effciency Agrobacterium-mediated transformation of Norway spruce (Picea abies) and loblolly pine (Pinus taeda). Plant Mol Biol 39: 407-416

Yeung EC (1995) Structural and developmental patterns in somatic embryogenesis. In: Thorpe TA (ed.) In Vitro Embryogenesis in Plants. Kluwer Academic Publishers, The Netherlands, pp 205-248 
Table 1 European chestnut lines used for cryopreservation

\begin{tabular}{ll}
\hline Line & Gene constructs in transgenic lines \\
\hline C12-H1/wt & Wild-type \\
C12-H1/C58C1-1 & pBI121 with nptII and uidA \\
C12-H1/pUbi-71 & pUbiGUSINT with nptII and uidA \\
C12-H1/p35S-10 & p35SGUSINT with nptII and uidA
\end{tabular}


Table 2 Effect of the genotype on the transformation efficiency of chestnut embryogenic cultures. Values represent means $\pm \mathrm{SE}$ of 6 replicates with 10 explants/replicate.

\begin{tabular}{ccc}
\hline Line & Kanamycin resistant (\%) & Transformation efficiency (\%) \\
\hline C12-H1 & $25.0 \pm 7.7$ & $21.7 \pm 6.7$ \\
CI-1 & $11.7 \pm 1.7$ & $3.3 \pm 2.1$ \\
CI-3 & $10.0 \pm 3.7$ & $5.0 \pm 2.2$ \\
CI-6 & $1.7 \pm 1.7$ & $1.7 \pm 1.7$ \\
CI-7 & $41.7 \pm 6.1$ & $33.8 \pm 7.8$ \\
CI-9 & $10.0 \pm 3.7$ & $10.0 \pm 3.7$ \\
HV-Z2 & $13.3 \pm 6.8$ & $1.7 \pm 1.7$ \\
\hline
\end{tabular}




\section{Figure Legends}

Figure 1. Effect of the type of explant on the transformation efficiency of chestnut embryogenic line CI-7. Values represent means \pm SE. Percentage values followed by the same letter are not significantly different at the $P=0.05$ level.

Figure 2. Embryo recovery frequencies of chestnut somatic embryo clumps of untransformed (wt) and transgenic lines (C58C1-1, pUbi-71 and p35S-10) after preculture on sucrose-containing medium and exposure to PVS2 solution for $60 \mathrm{~min}$, with $(\mathrm{LN}+)$ or without (LN-) subsequent immersion in liquid nitrogen. Untreated controls received neither sucrose preculture nor PVS2 treatment. Values represent means $\pm \mathrm{SE}$.

Figure 3. Transgenic plant derived from a transgenic, cryopreserved somatic embryo, line $\mathrm{C} 12-\mathrm{H} 1 / \mathrm{p} 35 \mathrm{~S}-10$.

Figure 4. Southern-blot analysis from DNA of the uidA gene in the transgenic cryopreserved line C12-H1/p35S-10. a: untreated control; b: PVS2 (LN-); c: PVS2 $(\mathrm{LN}+)$. 
Figure 1

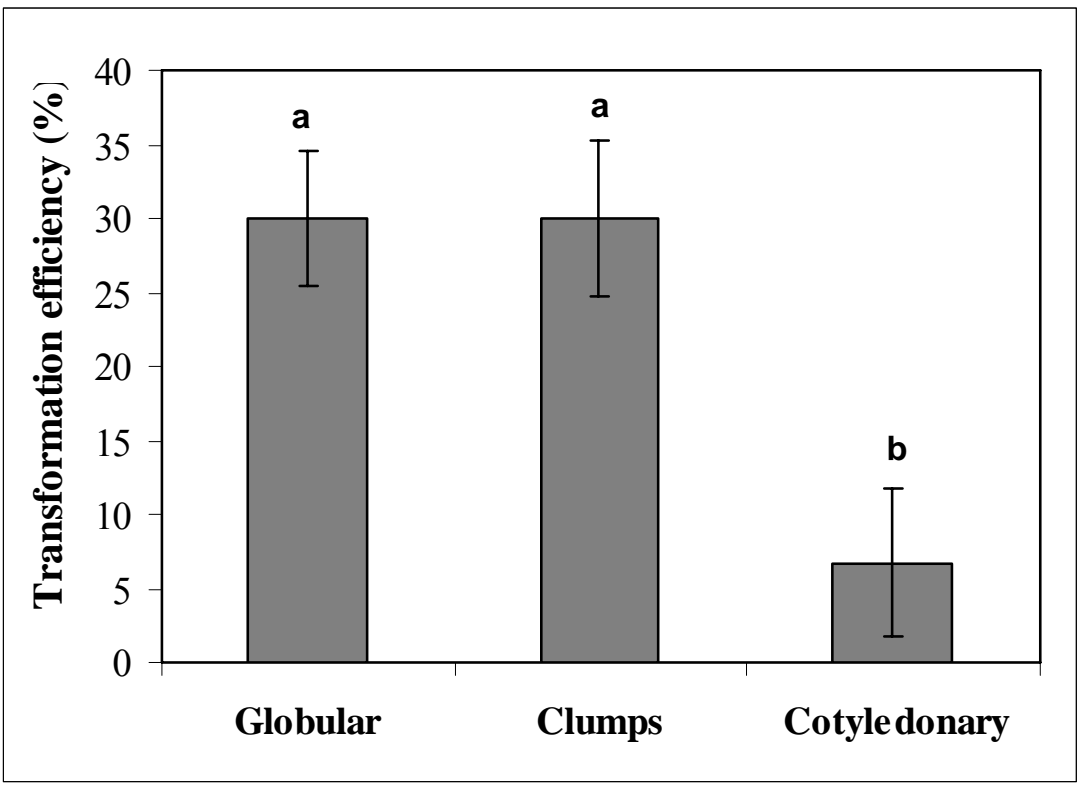


Figure 2

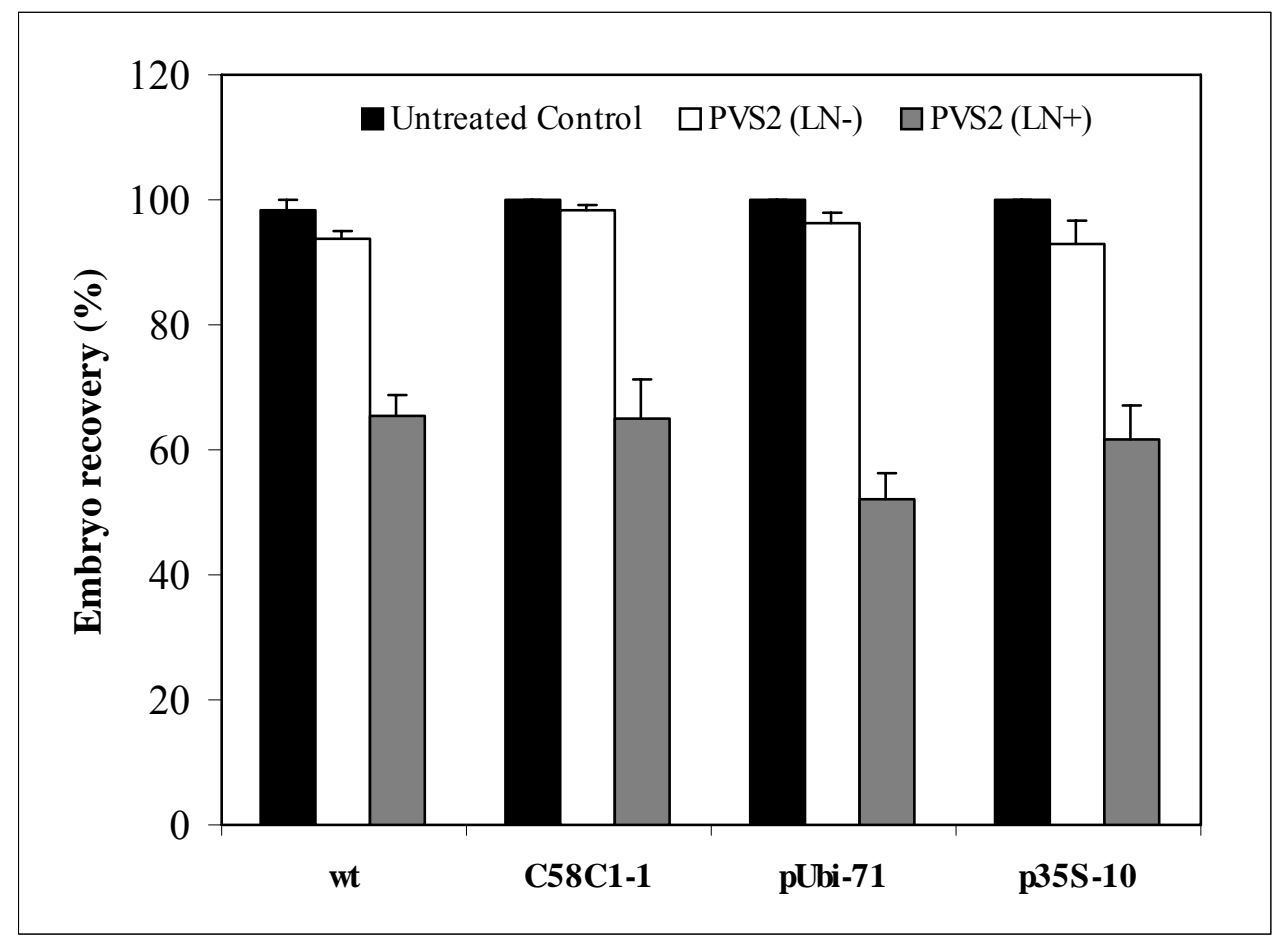


Figure 3

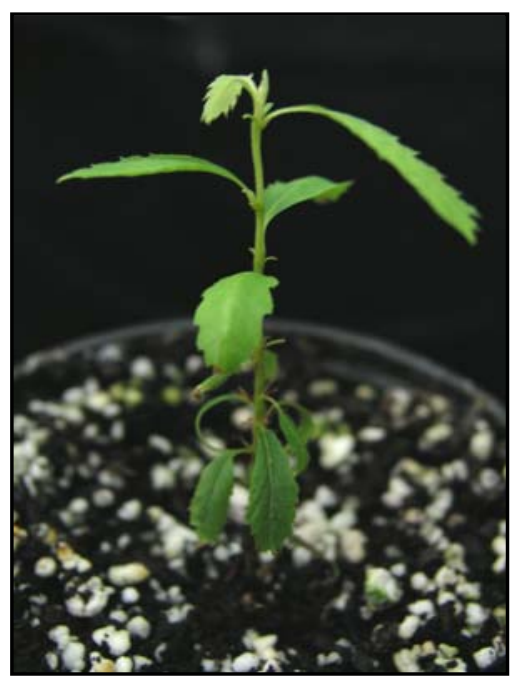


Figure 4

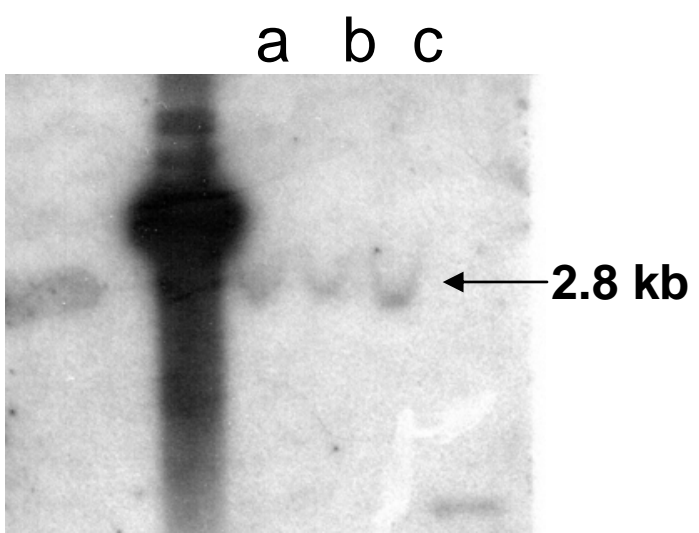

